\title{
Efecto de la inoculación con hongos micorrízicos arbusculares (HMA) y humus de lombriz en el establecimiento de Cenchrus purpureus (Schumach.) Morrone cv. Cuba CT-115
}

\author{
Effect of inoculating the arbuscular mycorrhizal fungi (AMF) and worm humus in \\ establishment of Cenchrus purpureus (Schumach.) Morrone cv. Cuba CT-115
}

\author{
Lázaro J. Ojeda Quintana ${ }^{1}$, Osvaldo Arteaga Rodríguez², \\ Lino A. Escobar Escobar ${ }^{1}$, Anaisa López Milián ${ }^{3}$
}

\begin{abstract}
RESUMEN
La investigación se realizó en la Estación Experimental de Suelos Escambray, ubicada en el poblado de Barajagua, municipio de Cumanayagua, provincia de Cienfuegos, región centro sur de Cuba. El objetivo del estudio fue determinar el rendimiento y calidad del forraje de Cenchrus purpureus Sch. cv. Cuba CT-115 inoculado con diferentes especies de hongos micorrízicos arbusculares (HMA) y la aplicación de humus de lombriz, en un diseño de bloques al azar con seis tratamientos y tres repeticiones. Se determinó el rendimiento de biomasa, contenido de nitrógeno y fósforo foliar, proteína bruta y el Índice de Eficiencia Micorrízica. El rendimiento de biomasa fue superior con la fertilización basal de NPK, mientras que con el humus de lombriz, las especies de HMA Funneliformis mosseae, Rhizoglomus irregulare y Glomus cubense superaron al testigo. La eficiencia micorrízica más alta estuvo en Funneliformis mosseae. El nitrógeno foliar fue mayor en la fertilización mineral, aunque sin diferencias con Funneliformis mosseae y Rhizoglomus irregulare, y estos dos tratamientos, a su vez, no difirieron del humus y de Glomus cubense. El contenido más bajo fue en el testigo. El fósforo no se difirió estadísticamente entre los tratamientos. El mayor porcentaje de proteína bruta se encontró en el tratamiento con la fertilización mineral, sin diferencias de Rhizoglomus irregulare y Funneliformis mosseae, mientras que el valor más bajo se obtuvo en el testigo. Se concluye que la inoculación con HMA superó en los indicadores evaluados al testigo, lo que representa para los productores una opción de menor costo económico y protección ambiental en función de incrementar el rendimiento y calidad del Cenchrus purpureus Sch. cv. Cuba CT-115.
\end{abstract}

Palabras clave: biofertilizantes, gramínea forrajera, rendimiento, proteína bruta.

\begin{abstract}
The investigation was carried out in the Experimental Station of Soils "Escambray", located in the town of Barajagua, municipality of Cumanayagua, in the county of Cienfuegos, region center south of Cuba. The study aims were to determine the yield and quality of the forage of Cenchrus purpureus $S c h . c v$. Cuba CT-115, inoculated with different arbuscular mycorrhizal fungi (AMF) and the application of worm humus. An experimental block design with completely randomized, six treatments, and three repetitions was used. It was determined the yield of biomass, nitrogen, and phosphorus content in the foliate, gross protein and Efficiency mycorrhizal Index. The yield of biomass was superior with the basal fertilization of NPK, while the worm humus, the species of HMA Funneliformis mosseae, Rhizoglomus irregulare, and Glomus cubense overcame the witness. The higher mycorrhizal efficiency was in the specie Funneliformis mosseae.

Regarding nitrogen content had the more significant in the mineral fertilization, although without differences with Funneliformis mosseae and Rhizoglomus irregulare, these two treatments, in turn, did not differ from the worm humus, and of Glomus cubense. The lowest content was in the witness; contrarily, the phosphorus content did not differ statistically among the treatments. The biggest percentage of gross protein was in the treatment with the mineral fertilization, without differences of Rhizoglomus irregulare and Funneliformis mosseae. The lowest content was in the control group. It is concluded that the inoculation with AMF it overcomes the one overcame in the indicators evaluated the witness, what represents one option for the producers, a smaller economic cost and environmental protection in function of increasing the yield and quality of the Cenchrus purpureus Sch. cv. Cuba, CT-115.

Keywords: biofertilizers, grass forage, yield, gross protein.
\end{abstract}

\footnotetext{
Centro Universitario Municipal Cumanayagua, Universidad de Cienfuegos, Cuba.

Unidad Científico Técnica de Base Suelos, Barajagua, Cienfuegos, Cuba.

Facultad Ciencias Agrarias, Universidad de Cienfuegos, Cuba.

* Autor por correspondencia: joberoverde@azurina.cult.cu
}

Fecha de Recepción: 9 de Noviembre, 2019.

Fecha de Aceptación: 26 de Febrero, 2020. 


\section{Introducción}

La producción ganadera en todo el mundo es un sector grande, dinámico y en fuerte crecimiento. Esto sucede en un ambiente en el cual muchos bienes comunes se encuentran amenazados: el sustento de vida de sectores pobres, la seguridad alimentaria, los recursos naturales, el medio ambiente, la salud humana y animal. Obviamente esta situación requiere de políticas sectoriales, instituciones y mejores reglamentos para enfrentar conflictos y desventajas que puedan resultar de un desarrollo del sector (Friedrich, 2014).

Los pastos y forrajes, de acuerdo con Herrera (2014), constituyen la principal fuente de alimentación para el ganado vacuno, y ello se debe a la posibilidad de cultivarlos todo el año. Además, si son manejados correctamente llegan a producir altos rendimientos de aceptable calidad capaces de cubrir la demanda diaria que requiere el bovino.

En Cuba, la base alimentaria en los sistemas de producción de leche, la constituyen los pastos y forrajes, que representan más del $90 \%$ de la materia seca de la dieta de los bovinos y permiten su utilización durante todo el año. Sin embargo, se han encontrado problemas graves de deterioro de los pastizales, aproximadamente entre un $7-12 \%$ de la superficie agrícola de la ganadería cubana con un descenso importante en los indicadores de producción y económicos (Montejo, 2016).

La especie forrajera más usada en Cuba, después de la caña de azúcar, es Pennisetum purpureum. La yerba elefante Cuba CT-115 ha sido liberada para la producción de forraje en pie y pastoreo directo, por su baja altura y aceptable rendimiento (Martínez y Herrera, 2006).

El uso de biofertilizantes es una de las técnicas empleadas para obtener elevados rendimientos en los cultivos, sin causarle daños al ambiente. La inclusión de hongos micorrízicos arbusculares (HMA) en los sistemas de biofertilización para los pastos y cultivos forrajeros puede ser una vía efectiva para mejorar su rendimiento y valor nutritivo, pues los beneficios de estos microorganismos en los agroecosistemas de pastizales están muy ligados al aumento de la superficie de absorción de las raíces y, en consecuencia, a la mejora de la eficiencia de la utilización de los nutrientes por las plantas (Verbruggen et al., 2013).

Su uso racional como inoculantes en la agricultura representa una reducción sustancial de agroquímicos, tales como fertilizantes y productos fitosanitarios, por lo que se les reconoce un gran potencial en el contexto de la agricultura sostenible (Azcón y Barea, 2015).

El objetivo de este trabajo fue determinar la respuesta del rendimiento y calidad de la biomasa, así como el contenido de fósforo y nitrógeno de Cenchrus purpureus (Schumach.) Morrone cv. Cuba CT-115 inoculado con diferentes especies de HMA.

\section{Materiales y métodos}

La investigación se realizó en áreas experimentales de campo de la Unidad Científica de Base Suelos, ubicada en el poblado de Barajagua, municipio de Cumanayagua, provincia de Cienfuegos, región centro sur de Cuba, cuyas coordenadas son $22^{\circ} 09^{\prime}$ de latitud norte y $80^{\circ} 12^{\prime}$ de longitud oeste, a $60 \mathrm{msnm}$. El suelo se clasifica como Pardo Grisáceo (Hernández et al., 2015), con un pH en agua 5,68, Norma Cubana ISO10390 (ONN, 1999a); materia orgánica de 1,78\%, Norma Cubana ISO-51 (ONN, 1999b); $\mathrm{P}_{2} \mathrm{O}_{5} 3.62$ y $\mathrm{K}_{2} \mathrm{O} 8,35 \mathrm{mg} / 100 \mathrm{~g}$ de suelo respectivamente, Norma Cubana ISO-52 (ONN, 1999c). El humus de lombriz mostró un 0,99, 0,47 y 0,36\% de N, P y $\mathrm{K}$, respectivamente, y $21,11 \%$ de materia orgánica.

Se estudió la inoculación de Cenchrus purpureus (Schumach.) Morrone cv. Cuba CT-115 con tres especies de HMA, la aplicación de humus de lombriz, fertilización mineral y un testigo de referencia, para un total de 6 tratamientos, todos en un diseño de bloques al azar con 3 réplicas:

\section{Funneliformis mosseae.}

2. Glomus cubense.

3. Rhizoglomus irregulare.

4. Humus de lombriz.

5. NPK en plantación.

6. Testigo.

Las especies de HMA evaluadas fueron Funneliformis mosseae Nicol. y Gerd. Walker y Schüßler (Schüßler y Walker, 2011), Glomus cubense Y. Rodr. y Dalpé (Rodríguez et al., 2011) y Rhizoglomus irregulare, N.C. Schenck y G.S. Sm. Sieverd., G.A. Silva y Oehl (Sieverding et al., 2014), procedentes de la colección de especies de HMA del Instituto Nacional de Ciencias Agrícolas (INCA). 
Se conformaron parcelas de $8,40 \mathrm{~m}^{2}$, con una separación entre sí de 0,5 m. Las estacas del CT-115 tenían $25 \mathrm{~cm}$ de longitud y se plantaron en el fondo del surco de forma horizontal. Como unidad de evaluación se tomaron los 2 surcos centrales, descartando los bordes, para un área de $2,10 \mathrm{~m}^{2}$ por parcela.

La inoculación de Cenchrus purpureus (Schumach.) Morrone cv. Cuba CT-115 se realizó mediante la técnica de recubrimiento de las semillas en el momento de la plantación. Se utilizó inóculo certificado con una pureza de 30 esporas g de suelo $^{-1}$ como promedio.

El NPK y el humus de lombriz se aplicaron sólo una vez, en el fondo del surco durante la plantación.El primero, a razón de $45 \mathrm{~kg} \mathrm{ha}^{-1}$ de nitrógeno, $40 \mathrm{~kg} \mathrm{ha}^{-1}$ fósforo y $120 \mathrm{~kg} \mathrm{ha}^{-1} \mathrm{de}$ potasio, con los portadores minerales Nitrato de amonio; y el humus de lombriz a 6t/ha.

El corte de establecimiento se efectuó a los 133 días, de forma manual a $5 \mathrm{~cm}$ sobre el nivel del suelo. En ese momento se cuantificó la masa verde en el área evaluable, y se tomaron 200 gramos de material verde para calcular en el laboratorio la materia seca total (\%) y el porcentaje foliar de nitrógeno y fósforo.

El rendimiento de biomasa $\left(\mathrm{t} / \mathrm{ha}^{-1}\right)$ y contenido de proteína bruta (\%) se determinaron de acuerdo a las fórmulas que siguen:

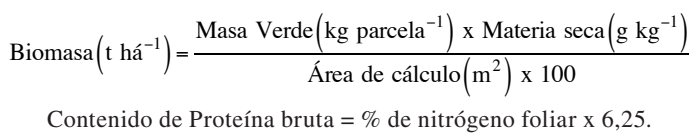

Fue calculado el Índice de Eficiencia Micorrízica (IEM), según la fórmula de Siquiera y Franco (1987).

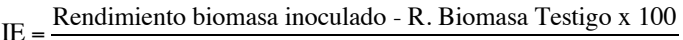
R. Biomasa Testigo

El comportamiento de la pluviometría fue de $355 \mathrm{~mm}$ entre los cinco meses, con cero precipitaciones en el mes de diciembre, mientras la temperatura estuvo en $24,5^{\circ} \mathrm{C}$ como promedio del período.

Todos los caracteres cumplieron los supuestos de normalidad y homogeneidad de varianza, por lo cual se procedió a efectuar un ANOVA de clasificación simple. Para la discriminación de medias se utilizó la prueba de comparación múltiple de Tukey ( $\mathrm{p} \leq 0,05)$, en los casos en que el ANOVA resultó significativo. Como herramienta de uso, el programa estadístico SPSS (versión 15.0).

\section{Resultados y discusión}

La brotación de Cenchrus purpureus (Schumach.) Morrone cv. Cuba CT-115 se produjo de manera uniforme entre los 10-12 días posteriores a la plantación. No hubo afectaciones de plagas y enfermedades en ninguna de las variantes evaluadas durante el ciclo del cultivo.

La Figura 1 refleja el rendimiento de biomasa de Cenchrus purpureus (Schumach.) Morrone cv. Cuba CT-115 en el corte de establecimiento. El mayor rendimiento se encontró en el tratamiento 5 (NPK), con 10,38 $\mathrm{t} / \mathrm{ha}^{-1}$, que difirió estadísticamente $(\mathrm{p} \leq 0,05)$ del resto de las variantes. En el tratamiento con la aplicación del humus de lombriz $(7,04$ t/ha-1), las especies Funneliformis mosseae $\left(6,97 \mathrm{t} / \mathrm{ha}^{-1}\right)$, Rhizoglomus irregulare, $\left(6,61 \mathrm{t} / \mathrm{ha}^{-1}\right)$ y Glomus cubense $\left(6,10 \mathrm{t} / \mathrm{ha}^{-1}\right)$ no difirieron estadísticamente entre sí. Por su parte, el testigo $\left(3,43 \mathrm{t} / \mathrm{ha}^{-1}\right)$ fue diferente estadísticamente de todos los tratamientos con el valor más bajo de rendimiento.

Resultó evidente el efecto favorable de la inoculación con las micorrizas y la aplicación del humus de lombriz en el incremento de forraje con relación al testigo. Este resultado presupone concebir variantes orgánicas y biológicas que pudieran suplir la ausencia de fertilizantes minerales, dada su carestía y, además, la necesidad de contribuir a la protección del medio ambiente.

El rendimiento alcanzado fue en condiciones de secano y sin fertilización mineral, con la excepción de la variante N.P.K. Estos son factores que, según Nava et al., (2013), pueden disminuir la producción de forraje.

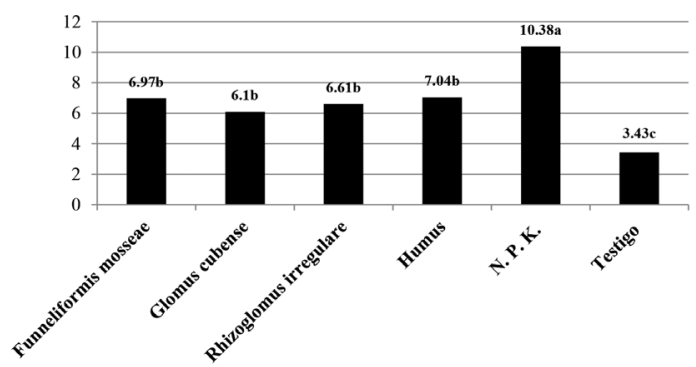

Figura 1. Rendimiento de biomasa seca total $\left(\mathrm{t} / \mathrm{ha}^{-1}\right)$ del CT115. Barras seguidas de distinta letra presentan diferencias significativas según Test Tukey $(\mathrm{p} \leq 0,05)$. 
Martínez y Herrera, (2006) señalaron que en Pennisetum se puede llegar a tener rendimientos de $12.4 \mathrm{t} / \mathrm{ha}$ de materia seca por cortes. El rendimiento obtenido quedó por debajo de este reporte, incluso en la variante fertilizada, lo que pudo deberse al comportamiento inferior de las precipitaciones en el período de establecimiento.

Mujica y Molina (2017) evaluaron en un suelo Pardo Ócrico Carbonatado, con pH de 7,2 y 1,02\% de materia orgánica, el efecto de R. intraradices y el estimulador del crecimiento vegetal FitoMas ${ }^{\circledR}$-E en la producción de biomasa de Pennisetum purpureum Sch. cv. Cuba CT-115. Se encontró que el tratamiento con R. intraradices y FitoMas (B) -E $+25 \mathrm{~kg} \mathrm{ha}^{-1}$ de urea alcanzó valores de 5,7 $\mathrm{t} \mathrm{ha}^{-1}$ masa seca en un corte. Con la aplicación de estos productos se incrementó la producción de biomasa del CT-115. A pesar de haber aplicado un estimulador del crecimiento vegetal y urea, los resultados alcanzados por estos autores no superaron el rendimiento de las variantes inoculadas indistintamente con HMA y humus de lombriz.

Caballero et al., (2016), en un suelo Ferralítico Rojo cuarcítico (FRC), de pH ácido (4,5-5,2) y contenido de materia orgánica de 2 a $5 \%$, determinaron el rendimiento y la calidad de cinco accesiones de C. purpureus (King grass, OM-22, CT169, Taiwán morado y CT-115). El comportamiento del rendimiento y la calidad de las accesiones contribuyeron a demostrar las potencialidades productivas del género Cenchrus en los suelos FRC.

El Índice de Eficiencia Micorrízica de las especies expresa, en términos porcentuales, el efecto en el incremento de la productividad del pasto con respecto al tratamiento sin inocular (testigo). Se se comportó en Funneliformis mosseae con un (103,2\%), Glomus cubense (92,70\%) y Rhizoglomus irregulare $(77,84 \%)$. Este indicador debe ser tomado en cuenta en cualquier análisis de la efectividad hongo/planta porque refleja cuánto puede influir la simbiosis en la planta hospedera en relación con un testigo. Se aprecia que Funneliformis mosseae y Rhizoglomus irregulare alcanzaron la mayor eficiencia, lo que se corresponde además con los mayores rendimientos de biomasa obtenidos.

En relación con el efecto de inocular HMA, aunque se reconoce que la respuesta de los pastos suele variar en función de muchos factores debido a que la ecofisiología de estos microorganismos en los agroecosistemas de pastizales es bastante compleja, varios autores coinciden en que la introducción de especies seleccionadas, adaptadas a las condiciones ambientales y con un alto nivel de compatibilidad funcional y ecológica para el sistema suelo-planta, puede ser una opción de manejo en los casos en que las cepas residentes no sean lo suficientemente efectivas para producir una respuesta agronómica (Melo de Miranda et al., 2008).

Furrazola et al. (2015) determinaron en áreas de la cuenca pecuaria El Tablón, perteneciente a la UCTB de Suelos Cienfuegos, la presencia de HMA nativas asociadas a cuatro pasturas establecidas. Se evaluaron las gramíneas Megathyrsus maximuss vc. likoni, Pennisetum purpureum vc. King grass, Brachiaria decumbens vc. CIAT-606 y Cynodon nlemfuensis vc. Jamaicano. Todas las especies mostraron colonización micorrízica arbuscular. Lla mayor colonización se obtuvo en Brachiaria decumbens vc. CIAT-606, con $66,27 \%$, mientras que las raicillas de Pennisetum purpureum vc. King grass mostraron los menores índices, con $48,80 \%$. Este comportamiento de las micorrizas nativas sugiere la necesidad de evaluar especies comerciales.

González et al. (2015) encontraron en un suelo Vertisol Pélico respuesta favorable del rendimiento de los pastos a la inoculación con micorrizas introducidas, en relación con las cepas residentes. La especie con mejor comportamiento fue Rhizoglomus intraradices. En los suelos Ferralítico Rojo Lixiviado y Gley Nodular Ferruginoso, Glomus cubense y Funneliformis mosseae respondieron mejor. Tales resultados revelaron mayor efectividad de las cepas introducidas. Estos autores consideran que las micorrizas resultan una alternativa para mejorar la productividad de las especies forrajeras.

García et al. (2014) evaluaron el comportamiento productivo de cuatro cultivares de Pennisetum purpureum (King-grass, Cuba CT-169, Cuba CT-115 y Taiwán morado), en un suelo Pardo Grisáceo de Las Tunas, Cuba, con pH 5,7 y materia orgánica $1,9 \%, \mathrm{P}_{2} \mathrm{O}_{5} 2,01$ y $\mathrm{K}_{2} \mathrm{O} 4,02 \mathrm{mg} / 100 \mathrm{~g} /$ suelo, respectivamente. Los resultados mostraron un rendimiento de Cuba CT-115 de 4,2 t/ha ${ }^{-1}$ en el período menos lluvioso, muy por debajo del alcanzado en este trabajo con condiciones similares de suelo y precipitaciones.

La Figura 2 refleja el contenido foliar de nitrógeno y fósforo de Cenchrus purpureus CT115. Se aprecia que Rhizoglomus irregulare mostró el mayor valor absoluto de nitrógeno, aunque sin 


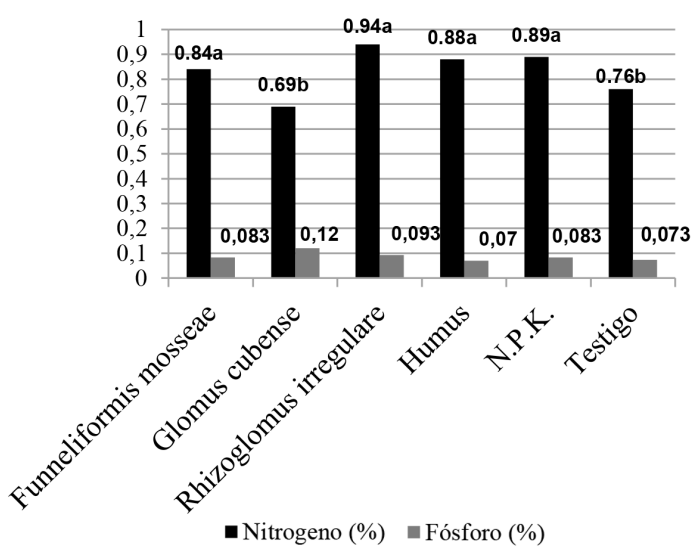

Figura 2. Contenido de nitrógeno y fósforo en el CT-115. Barras seguidas de distinta letra presentan diferencias significativas según Test Tukey $(\mathrm{p} \leq 0,05)$.

diferir del NPK, humus y Funneliformis mosseae. El menor contenido de nitrógeno estuvo en el testigo y en la especie de HMA Glomus cubense.

Incrementar el contenido de $\mathrm{N}_{2}$ en la planta, según (Borges et al., 2012), estimula el crecimiento y aumenta la utilización de carbohidratos disponibles para la formación de células y de protoplasma, sin incrementar el grosor de la pared celular. De este modo, se reducen los tenores de fibra y lignina y aumenta la digestibilidad y el valor nutritivo del pasto. Este aspecto resulta muy ventajoso para la utilización del CT-115 como alternativa forrajera o de pastoreo en función de mejorar la calidad del pasto para el consumo animal.

El contenido de fósforo no evidenció diferencias estadísticas entre los tratamientos $(\mathrm{p} \leq 0,05)$. Sin embargo, los tratamientos Glomus cubense y Rhizoglomus irregulare mostraron los mayores tenores de fósforo, mientras que el testigo y el humus de lombriz los más bajos. Resulta importante que el contenido de ambos elementos se favoreciera por la acción de los HMA con relación al testigo, lo que puede ofrecer una alternativa para productores de bajos insumos en cuanto a mejorar la nutrición de esta especie de pasto.

Los HMA juegan un papel vital en la toma del P presente en los suelos, principalmente en las zonas tropicales donde las cantidades asimilables de este elemento son bajas. El mecanismo para incrementar la absorción vía HMA se desarrolla a partir de la capacidad de explorar un mayor volumen de sustrato y aumentar la capacidad absorbente de las raíces (interceptación) y por difusión, que es el transporte de nutrientes a lo largo de un gradiente de concentración. Además, las micorrizas pueden acelerar la absorción de P no disponible para las plantas por la interacción entre los exudados radicales de algunas especies de plantas que son capaces de solubilizar P (Shibata y Yano, 2003).

La respuesta de las variantes inoculadas con las micorrizas pudo estar dada por lo expresado por (Yang et al., 2014), al señalar que los HMA son componentes esenciales de la rizosfera de los pastizales, cuyas plantas permanecen asociadas mediante una red de hifas interconectadas que incrementan el volumen de suelo que exploran las raíces y facilitan la absorción de los nutrientes y el agua, entre otras funciones.

La Figura 3 refleja el contenido de proteína bruta (PB) en los diferentes tratamientos evaluados en el trabajo. Se aprecia que el mayor contenido de PB se encontró en la variante que recibió la fertilización mineral, sin diferencias de Rhizoglomus irregulare y Funneliformis mosseae, que no difirió de Glomus cubense y el humus de lombriz. El valor más bajo de proteína bruta se observó en el testigo, con diferencias del resto de las variantes. Los resultados alcanzados indican el efecto favorable de los HMA al mejorar el contenido de proteína bruta de la biomasa de este pasto.

Las especies de pastos del género Pennisetum son las más promisorias para la ganadería, sus niveles de proteína oscilan entre 3\% y 20\% (Suárez, 2016). Los valores alcanzados en el trabajo se enmarcaron entre 4,10 y $5,73 \%$.

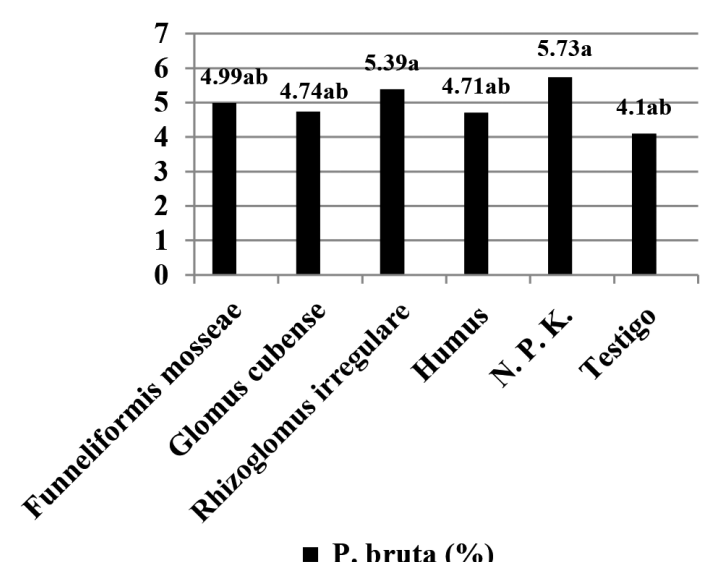

- P. bruta (\%)

Figura 3. Porcentaje de proteína bruta del CT-115. Barras seguidas de distinta letra presentan diferencias significativas según Test Tukey $(\mathrm{p} \leq 0,05)$. 


\section{Conclusiones}

El rendimiento de biomasa fue superior con la fertilización basal de NPK, mientras que con el humus de lombriz, las especies de HMA Funneliformis mosseae, Rhizoglomus irregulare y Glomus cubense superaron al testigo. El mayor Índice de Eficiencia Micorrízica estuvo en Funneliformis mosseae.

El nitrógeno foliar fue mayor en la fertilización mineral, aunque sin diferencias con Funneliformis mosseae y Rhizoglomus irregulare. Estos dos tratamientos, a su vez, no difirieron del humus y de Glomus cubense. El contenido más bajo fue en el testigo. El fósforo no se diferenció estadísticamente entre los tratamientos.

El mayor porcentaje de proteína bruta se encontró en el tratamiento con la fertilización mineral, sin diferencias de Rhizoglomus irregulare y Funneliformis mosseae. El valor más bajo de proteína bruta se obtuvo en el testigo.

\section{Literatura Citada}

Azcón, C.; Barea, J.M.

2015. Nutrient cycling in the mycorrhizosphere. In: Gianfreda, L. (Guest Editor) Biogeochemical processes in the rhizosphere and their influence on plant nutrition. Journal of Soil Science and Plant Nutrition, 15: 372-396.

Borges, J.A.; Barrios, M.; Escalona, O.

2012. Efecto de la fertilización orgánica e inorgánica sobre variables agroproductivas y composición química del pasto estrella (Cynodon nlemfuensis). Zootecnia Tropical, 30 (1): 17-26.

Caballero, A.; Martínez, R.O., Hernández, M.; Navarro, M. 2016. Caracterización del rendimiento y la calidad de cinco accesiones de Cenchrus purpureus (Schumach.) Morrone. En Pastos y Forrajes, 39 (2): 94-101.

Friedrich, T.

2014. Producción de alimentos de origen animal. Actualidad y perspectivas. Revista Cubana de Ciencia Agrícola, 48 (1): 5-6.

Furrazola, E.; Ojeda, L.; Consuelo H.

2016. Colonización micorrízica y especies de hongos micorrizógenos arbusculares en gramíneas de la cuenca pecuaria El Tablón, Cienfuegos, Cuba. Cuban Journal of Agricultural Science, 50 (2): 321-331.

García, L.; Mesa, A.; Hernández, M.

2014. Potencial forrajero de cuatro cultivares de Pennisetum purpureum en un suelo Pardo de Las Tunas. Pastos y Forrajes, 37 (4): 413-419.

González, P.J.; Ramírez, J.F. Rivera, R.; Hernández, A., Plana,

R.; Crespo, G.; Flores, P.R.; Rosales, R.S.

2015. Manejo de la inoculación micorrízica arbuscular para el establecimiento, mantenimiento y recuperación de pastizales. En: Memoria del V Congreso de Producción Animal Tropical, 16-20 de noviembre. Palacio de Convenciones, La Habana, Cuba.

Herrera, R.

2014. Algunos aspectos que pueden influir en el rigor y veracidad del muestreo de pastos y forrajes. Revista de investigación y difusión científica agropecuaria, 18 (2): 7-26.

Hernández, A.; Pérez, J.M.; Bosch, D.; Castro, N.

2015 Clasificación de los suelos de Cuba. Mayabeque, Ediciones INCA, Instituto de Suelos. Cuba. 91 p.

Martínez, R.O. y Herrera, R.S.

2006. Empleo del Cuba CT-115 para solucionar el déficit de alimentos durante la seca. En: Producción y manejo de los recursos forrajeros tropicales. Eds. M.E. Velasco, A.
Hernández, R.A. Perezgrovas y B. Sánchez. Universidad Autónoma de Chiapas. México. P. 75.

Melo de Miranda, E.; Saggin Júnior, O.J.; Ribeiro da Silva, E. 2008. Seleção de fungos micorrízicos arbusculares para o amendoim forrageiro consorciado com braquiária. Pesq. Agropec. Bras., 43 (9): 1185-1191.

Mujica, Y.; Molina, L.

2017. Influencia de hongos micorrízicos arbusculares (Rhizoglomus intraradices) y un estimulador del crecimiento vegetal en Pennisetum purpureum Sch. cv. Cuba CT-115. Cultivos Tropicales, 38 (1): 131-137.

Nava, J.; Gutiérrez, E.; Herrera, R.; Zavala, F.; Olivares, E.; Treviño, J.; Bernal, H.; Valdés, C.

2013. Rendimiento y composición química del pasto CT-115 (Pennisetum purpureum) establecido a dos densidades y en dos fechas de siembra en Marín. Nuevo León, México. Revista Cubana de Ciencia, 47 (4): 419-424.

ONN.

1999a. Determinación de materia orgánica en suelo. NC-ISO 51. La Habana: Oficina Nacional de Normalización. La Habana, Cuba.

ONN.

1999b. Determinación del pH. NC-ISO 10390. La Habana: Oficina Nacional de Normalización. La Habana, Cuba. ONN.

1999c. Determinación de las formas móviles de potasio y fósforo. NC-ISO 52. La Habana: Oficina Nacional de Normalización. La Habana, Cuba.

Rodríguez, Y.; Dalpé, Y.; Séguin, S.; Fernández, K., Rivera, R. 2011. Glomus cubense sp. nov. an arbuscular mycorrhizal fungus from Cuba. Micotaxon, 118 (1): 337-47

Siqueira, J.O.; Colozzi-Filho, A.; Oliveira, E. de; Fernandes, A.B.; Florence, M.L.

1987. Micorrizas vesicular-arbusculares em mudas de cafeeiro produzidas no Sul do Estado de Minas Gerais. Pesq. Agrop. Bras., 22 (1): 31-38.

Schüßler, A.; Walker, C.

2011. Evolution of the Plant-Symbiotic Fungal Phyllum Glomeromycota. Evolution of fungi and fungal-like organisms. In: Pöggeler, S.; Wöstemeyer, j (ed.). The Mycota XIV. Heidelberg. Springer-Verlag. Berlin, Alemania. pp. 163-185.

Sieverding, E.; Alves da Silva, G.; Berndt, R.; Oehl, F.

2014. Rhizoglomus a new genus of the Glomeraceae. Mycotaxon, 129 (2): 373-86. 
Shibata, R.; Yano, K.

2003. Phosphorus acquisition from non-labile sources in peanut and pigeonpea with mycorrhizal interaction. Applied Soil Ecology, 24 (2): 133-141.

Valenciaga, D., Chongo, B., Herrera, R.S., Torres, V., Oramas, A., Cairo, J.G. y Herrea, M.

2009. Effect of regrowth age on the chemical composition of Pennisetum purpureum cv. CUBA CT-115. Cuban J. Agric. Sci.
Verbruggen, E.; van der Heijden, M.G.A.; Rillig, M.C.; Kiers, E.T. 2013. Mycorrhizal fungal establishment in agricultural soils: determining inoculation. New Phytologist. (4): 1104-9.

Yang, C.; Ellouze, W.; Navarro-Borrell, A.; Taheri, A.E.; Klabi, R.; Dai, M.

2014. Management of the Arbuscular Mycorrhizal Symbiosis In: Mycorrhizal Fungi: Use in Sustainable Agriculture and Land Restoration. Solaiman, Z. M.; Abbott, L.K.; Varma, A. Ed. Springer. Heidelberg, Berlín, Alemania. Pp. 89-118. 
